VOLUME 27 (2020) 114-126

DOI: $10.24330 /$ ieja.662993

\title{
GORENSTEIN $\pi[T]$-PROJECTIVITY WITH RESPECT TO A TILTING MODULE
}

\author{
M. Amini \\ Received: 13 February 2019; Revised: 11 October 2019; Accepted: 12 October 2019
}

Communicated by Abdullah Harmancı

\begin{abstract}
Let $T$ be a tilting module. In this paper, Gorenstein $\pi[T]$-projective modules are introduced and some of their basic properties are studied. Moreover, some characterizations of rings over which all modules are Gorenstein $\pi[T]$-projective are given. For instance, on the $T$-cocoherent rings, it is proved that the Gorenstein $\pi[T]$-projectivity of all $R$-modules is equivalent to the $\pi[T]$-projectivity of $\sigma[T]$-injective as a module.
\end{abstract}

Mathematics Subject Classification (2010): 13D07, 16D40, 18G25

Keywords: Cocoherent ring, dimension, Gorenstein module, tilting module

\section{Introduction}

Throughout this paper, $R$ is an associative ring with non-zero identity, all modules are unitary left $R$-modules. First we recall some known notions and facts needed in the sequel. Let $R$ be a ring and $T$ an R-module. Then

(1) We denote by ProdT (resp. F.ProdT), the class of modules isomorphic to direct summands of direct product of copies (resp. finitely many copies) of $T$.

(2) We denote by $A d d T$ (resp. F.AddT), the class of modules isomorphic to direct summands of direct sum of copies (resp. finitely many copies) of $T$.

(3) Following [3], a module $T$ is called tilting (1-tilting) if it satisfies the following conditions:

(a) $\operatorname{pd}(T) \leq 1$, where $p d(T)$ denotes the projective dimension of $T$.

(b) $\operatorname{Ext}^{i}\left(T, T^{(\lambda)}\right)=0$, for each $i>0$ and for every cardinal $\lambda$.

(c) There exists the exact sequence $0 \rightarrow R \rightarrow T_{0} \rightarrow T_{1} \rightarrow 0$, where $T_{0}, T_{1} \in \operatorname{Add} T$.

(4) By Copres $^{n} T$ (resp. F.Copres ${ }^{n} T$ ) and Copres $^{\infty} T$ (resp. F.Copres ${ }^{\infty} T$ ), we denote the set of all modules $M$ such that there exists exact sequences

$$
0 \longrightarrow M \longrightarrow T_{0} \longrightarrow T_{1} \longrightarrow \cdots \longrightarrow T_{n-1} \longrightarrow T_{n}
$$


and

$$
0 \longrightarrow M \longrightarrow T_{0} \longrightarrow T_{1} \longrightarrow \cdots \longrightarrow T_{n-1} \longrightarrow T_{n} \longrightarrow \cdots,
$$

respectively, where $T_{i} \in \operatorname{Prod} T\left(\operatorname{resp} . T_{i} \in \mathrm{F} . \operatorname{Prod} T\right)$, for every $i \geq 0$.

(5) A module $M$ is said to be cogenerated, by $T$, denoted by $M \in C o g e n T$, (resp. generated, denoted $M \in G e n T$ ) by $T$ if there exists an exact sequence $0 \rightarrow M \rightarrow T^{n}$ (resp. $T^{(n)} \rightarrow M \rightarrow 0$ ), for some positive integer $n$.

(6) Let $\mathcal{C}$ be a class of modules and $M$ be a module. A right (resp. left) $\mathcal{C}$ resolution of $M$ is a long exact sequence $0 \rightarrow M \rightarrow C_{0} \rightarrow C_{1} \rightarrow \cdots$ (resp. $\cdots \rightarrow C_{1} \rightarrow C_{0} \rightarrow M \rightarrow 0$ ), where $C_{i} \in \mathcal{C}$, for all $i \geq 0$. It is said that a module $M$ has right $\mathcal{C}$-dimension $n$ (briefly, $\mathcal{C} \cdot \operatorname{dim}(M)=n$ ) if $n$ is the least non-negative integer such that there exists a long exact sequence

$$
0 \longrightarrow M \longrightarrow C_{0} \longrightarrow C_{1} \longrightarrow \cdots \longrightarrow C_{n-1} \longrightarrow C_{n} \longrightarrow 0
$$

with $C_{i} \in \mathcal{C}$, for each $i \geq 0$. In particular, the $\operatorname{Prod} T$-dimension of $M$ is called $T$-injective dimension of $M$ and is denoted by T.i.dim( $M)$. Note that for any tilting module $M$, if $M \in \operatorname{Cogen} T$, then [6, Proposition 2.1] implies that $\operatorname{Cogen} T=\operatorname{Copres}^{\infty} T$. This shows that any module cogenerated by $T$ has an $\operatorname{Prod} T$-resolution. The $\operatorname{Prod} T$-resolutions and the relative homological dimension were studied by Nikmehr and Shaveisi in [6].

(7) For any homomorphism $f$, we denote by kerf and $i m f$, the kernel and image of $f$, respectively. Let $A$ and $M \in \operatorname{Cogen} T$ be two modules. We define the functor

where

$$
\mathcal{E}_{T}^{n}(A, M):=\frac{\operatorname{ker} \delta_{*}^{n}}{\operatorname{im} \delta_{*}^{n-1}}
$$

$$
0 \longrightarrow M \stackrel{\delta_{0}}{\longrightarrow} T_{0} \stackrel{\delta_{1}}{\longrightarrow} \cdots \stackrel{\delta_{n}}{\longrightarrow} T_{n} \longrightarrow \cdots
$$

$\operatorname{Prod} T$-resolution of $M$ and $\delta_{*}^{n}=\operatorname{Hom}\left(i d_{B}, \delta_{n}\right)$, for every $i \geq 0$. See [6,9] for more details.

(8) Let $M \in \operatorname{Cogen} T$ and $N$ be two modules. A similar proof to that of [7, Lemma 2.11] shows that $\mathcal{E}_{T}^{0}(N, M) \cong \operatorname{Hom}(N, M)$. Moreover, $\mathcal{E}_{T}^{1}(-, M)=$ 0 implies that $M \in \operatorname{Prod} T$, and if $M \in \operatorname{Gen} T$, then $\mathcal{E}_{T}^{1}(M,-)=0$ implies that $M \in \operatorname{Add} T$. It is clear that T.i.dim $(M)=n$ if and only if $n$ is the least non-negative integer such that $\mathcal{E}_{T}^{n+1}(A, M)=0$, for any module $A$, see $[6, \operatorname{Remark} 2.2]$ for more details. So, T.i.dim $(M)=n$ if and only if $\mathcal{E}_{T}^{n+i}(A, M)=0$ for every module $A$ and every $i \geq 1$. A module with zero $T$-injective dimension (resp. $T$-projective dimension) is called $T$-injective 
(resp. T-projective). A similar proof to that of [7, Proposition 2.3] shows that the definition of $\mathcal{E}_{T}^{n}(C, M)$ is independent from the choice of ProdTresolutions. For unexplained concepts and notations, we refer the reader to $[2,6,8]$.

(9) For a module $T$, we denote by $\pi[T]$, the full subcategory of modules whose objects are of the form $\frac{B}{A} \leq \frac{T^{I}}{A}$, for some cardinal $I$ and some modules $A \leq B \leq T^{I}$. Also, the full subcategory $\sigma[T]$ of modules subgenerated by a given module $T$ (see [10]).

(10) $G$ is called Gorenstein $\sigma[T]$-injective if there exists an exact sequence of $\sigma[T]$-injective modules

$$
\mathbf{A}=\cdots \longrightarrow A_{1} \longrightarrow A_{0} \longrightarrow A^{0} \longrightarrow A^{1} \longrightarrow \cdots
$$

with $G=\operatorname{ker}\left(A^{0} \rightarrow A^{1}\right)$ such that $\operatorname{Hom}(U,-)$ leaves this sequence exact whenever $U \in \operatorname{Pres}^{1} T$ with T.p.dim $(U)<\infty$ (see [9]).

(11) $M$ is said to be finitely cogenerated [2] if for every family $\left\{V_{k}\right\}_{J}$ of submodules of $M$ with $\bigcap_{J} V_{k}=0$, there is a finite subset $I \subset J$ with $\bigcap_{I} V_{k}=0$.

(12) $M$ is said to be finitely copresented if there is an exact sequence of $R$ modules $0 \rightarrow M \rightarrow E^{0} \rightarrow E^{1}$, where each $E^{i}$ is a finitely cogenerated injective module, see $[1,11,12]$.

Let $T$ be a tilting module. In this paper, we introduce the $\pi[T]$-projective modules, the $\pi[T]$-projective dimension and Gorenstein $\pi[T]$-projective modules.

Let $M \in$ GenT. Then, $M$ is called $\pi[T]$-projective if the functor $\mathcal{E}_{T}^{1}(M,-)$ vanishes on $\pi[T]$. Also, the $\pi[T]$-projective dimension of $M$ is defined to be

$$
\pi[T] \cdot p d(M)=\inf \left\{n: \mathcal{E}_{T}^{n+1}(M, N)=0 \text { for every } N \in \pi[T]\right\} .
$$

We define a module $G$ to be Gorenstein $\pi[T]$-projective ( $G T$-projective for short), if there exists an exact sequence of $\pi[T]$-projective modules

$$
\mathbf{B}=\cdots \longrightarrow B_{1} \longrightarrow B_{0} \longrightarrow B^{0} \longrightarrow B^{1} \longrightarrow \cdots
$$

with $G=\operatorname{ker}\left(B^{0} \rightarrow B^{1}\right)$ such that $\operatorname{Hom}(-, U)$ leaves this sequence exact whenever $U \in$ F.Copres ${ }^{1} T$ with T.i.dim $(U)<\infty$. In this paper, the $G T$-projective dimension of a module $G$ is denoted by $G T-p d(G)$.

In Section 2, we study some basic properties of the Gorenstein $\pi[T]$-projective modules. Recall that a ring $R$ is said to be cocoherent if every finitely cogenerated module is finitely copresented. So, $R$ is a cocoherent ring if and only if $\operatorname{Copres}^{0} R=$ Copres $^{1} R$. For more information about the cocoherent rings, we refer the reader 
to [5]. As a cogeneralization of this concept, we call a ring $R$ to be $T$-cocoherent if F.Copres ${ }^{0} T=$ F.Copres ${ }^{1} T$.

Section 3 is devoted to some characterizations of $T$-cocoherent rings over which all modules are Gorenstein $\pi[T]$-projective. For instance, it is proved that every module is Gorenstein $\pi[T]$-projective if and only if every $T$-injective module is $\pi[T]$ projective if and only if every $\sigma[T]$-injective module is Gorenstein $\pi[T]$-projective. Finally, we give a sufficient condition under which every Gorenstein $\pi[T]$-projective module is $\pi[T]$-projective.

\section{Gorenstein $\pi[T]$-projectivity}

We start with the following definition.

Definition 2.1. Let $T$ be a tilting module. Then

(1) $M$ is called $\pi[T]$-projective if $\mathcal{E}_{T}^{1}(M, N)=0$, for every $N \in \pi[T]$.

(2) Let $G \in \operatorname{Gen} T$. Then, $G$ is called Gorenstein $\pi[T]$-projective if there exists an exact sequence of $\pi[T]$-projective modules

$$
\mathbf{B}=\cdots \longrightarrow B_{1} \longrightarrow B_{0} \longrightarrow B^{0} \longrightarrow B^{1} \longrightarrow \cdots
$$

with $G=\operatorname{ker}\left(B^{0} \rightarrow B^{1}\right)$ such that $\operatorname{Hom}(-, U)$ leaves this sequence exact whenever $U \in$ F.Copres ${ }^{1} T$ with T.i. $\operatorname{dim}(U)<\infty$.

Remark 2.2. Let $T$ be a tilting module. Then

(1) $\mathcal{E}_{T}^{1}(N, M)=0$ for any $\pi[T]$-projective module $N$ and any $M \in \operatorname{Copres}^{0} T$.

(2) If $A \in \operatorname{Add} T$, then $A$ is $\pi[T]$-projective.

Lemma 2.3. Let $0 \rightarrow A \stackrel{f}{\rightarrow} B \stackrel{g}{\rightarrow} C \rightarrow 0$ be an exact sequence. Then

(1) If $A$ is $T$-injective and $A, B, C \in \operatorname{Cogen} T$, then $B=A \oplus C$.

(2) If $A \in$ F.Copres ${ }^{n} T$ and $C \in$ F.Copres ${ }^{n} T$, then $B \in$ F.Copres ${ }^{n} T$.

(3) If $C \in \mathrm{F}$.Copres ${ }^{n} T$ and $B \in \mathrm{F}$.Copres ${ }^{n+1} T$, then $A \in \mathrm{F}$.Copres ${ }^{n+1} T$.

(4) If $B \in \mathrm{F}$.Copres ${ }^{n} T$ and $A \in \mathrm{F}$.Copres ${ }^{n+1} T$, then $C \in \mathrm{F}$.Copres ${ }^{n} T$.

Proof. (1) If $A$ is $T$-injective and $A, B, C \in \operatorname{Cogen} T$, then we deduce that the sequence

$$
0 \longrightarrow \operatorname{Hom}(C, A) \stackrel{g^{*}}{\longrightarrow} \operatorname{Hom}(B, A) \stackrel{f^{*}}{\longrightarrow} \operatorname{Hom}(A, A) \longrightarrow \mathcal{E}_{T}^{1}(C, A)=0
$$

is exact. So, there exists $h: B \rightarrow A$ such that $h f=1_{A}$. 
(2) We prove the assertion by induction on $n$. If $n=0$, then the commutative diagram with exact rows

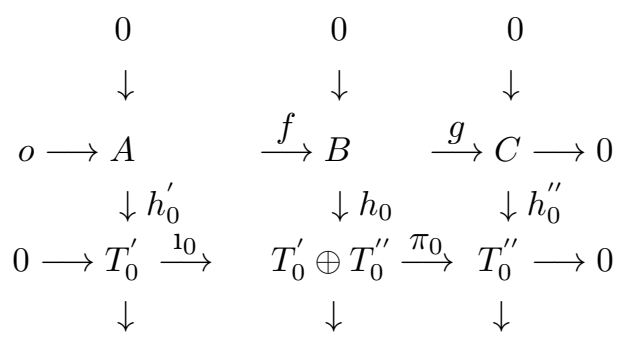

exists, where $T_{0}^{\prime}, T_{0}^{\prime \prime} \in$ F.Prod $T, i_{0}$ is the inclusion map, $\pi_{0}$ is a canonical epimorphism and $h_{0}=i_{0} h_{0}^{\prime}$ is endomorphism, by Five Lemma. Let $K_{1}^{\prime}=\operatorname{coker}\left(h_{0}^{\prime}\right)$, $K_{1}=\operatorname{coker}\left(h_{0}\right)$ and $K_{1}^{\prime \prime}=\operatorname{coker}\left(h_{0}^{\prime \prime}\right)$. It is clear that $\left(T_{0}^{\prime} \oplus T^{\prime \prime}\right) \in \operatorname{F} . \operatorname{Prod} T$ and $K_{1}^{\prime}, K_{1}^{\prime \prime} \in \mathrm{F}$.Copres ${ }^{n-1} T$; so, the induction implies that $K_{1} \in \mathrm{F}$.Copres ${ }^{n-1} T$. Hence $B \in$ F.Copres ${ }^{n} T$.

(3) Let $B \in$ F.Pres ${ }^{n+1} T$ and $C \in$ F.Pres ${ }^{n} T$, then the following commutative diagram with exact rows:

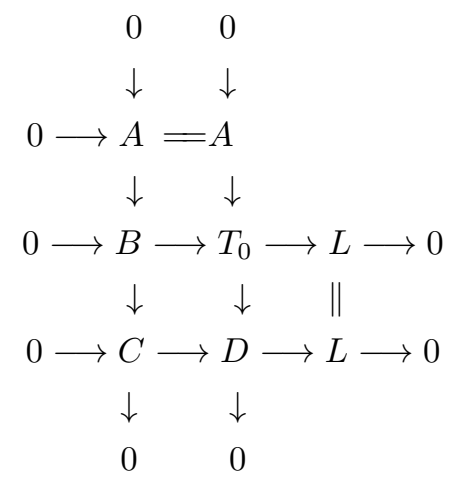

where $T_{0} \in$ F.Prod $T$ and $L \in$ F.Copres ${ }^{n} T$. By (2), $D \in$ F.Copres ${ }^{n} T$. So, we deduce that $A \in \mathrm{F}$. Copres $^{n+1} T$.

(4) Let $A \in$ F.Pres ${ }^{n+1} T$ and $B \in$ F.Pres ${ }^{n} T$, then the following commutative diagram with exact rows: 


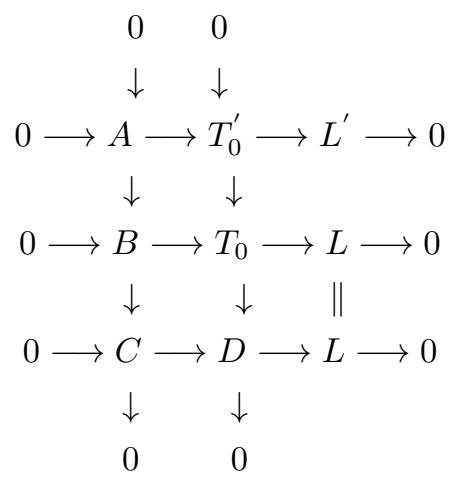

where $T_{0}, T_{0}^{\prime} \in$ F.Prod $T$ and $L \in$ F.Copres ${ }^{n-1} T$. Since $T_{0}^{\prime}$ is $T$-injective, we have that $T_{0}=T_{0}^{\prime} \oplus D$ By (1), and $D \in \operatorname{Cogen} T$. Thus for any $N \in \operatorname{Cogen} T$, we have

$$
\mathcal{E}_{T}^{1}\left(T_{0}, N\right)=\mathcal{E}_{T}^{1}\left(T_{0}^{\prime} \oplus D, N\right)=\mathcal{E}_{T}^{1}\left(T_{0}^{\prime}, N\right) \oplus \mathcal{E}_{T}^{1}(D, N)=0 .
$$

Hence $D \in$ F.ProdT. On the other hand, $L \in$ F.Copres ${ }^{n-1} T$. Therefore, we conclude that $C \in \mathrm{F}$. Copres $^{n} T$.

In the following theorem, we show that in the case of $T$-cocoherent rings, the existence of $\pi[T]$-projective complex of a module is sufficient to be Gorenstein $\pi[T]$ projective.

Theorem 2.4. Let $R$ be a $T$-cocoherent ring and $G \in \mathrm{Gen} T$ be a module. Then $G$ is Gorenstein $\pi[T]$-projective if and only if there is an exact sequence

$$
\mathbf{B}=\cdots \longrightarrow B_{1} \longrightarrow B_{0} \longrightarrow B^{0} \longrightarrow B^{1} \longrightarrow \cdots
$$

of $\pi[T]$-projective modules such that $G=\operatorname{ker}\left(B^{0} \rightarrow B^{1}\right)$.

Proof. $(\Rightarrow)$ : This is a direct consequence of definition.

$(\Leftarrow)$ : By definition, it suffices to show that $\operatorname{Hom}(\mathbf{B}, U)$ is exact for every module $U \in$ F.Copres ${ }^{1} T$ with T.i.dim $(U)=m<\infty$. To prove this, we use the induction on $m$. The case $m=0$ is clear. Assume that $m \geq 1$. Since $U \in \mathrm{F}$. $\operatorname{Copres}^{1} T$, there exists an exact sequence $0 \rightarrow U \rightarrow T_{0} \rightarrow I \rightarrow 0$ with $T_{0} \in$ F.Prod $T \subseteq$ F.Copres ${ }^{0} T$. Now, from the $T$-cocoherence of $R$ and Lemma 2.3, we deduce that $I, T_{0} \in$ F.Copres ${ }^{1} T$. Also, T.i.dim $(I) \leq m-1$ and T.i.dim $\left(T_{0}\right)=0$. Thus by Remark 2.2, the following short exact sequence of complexes exists: 


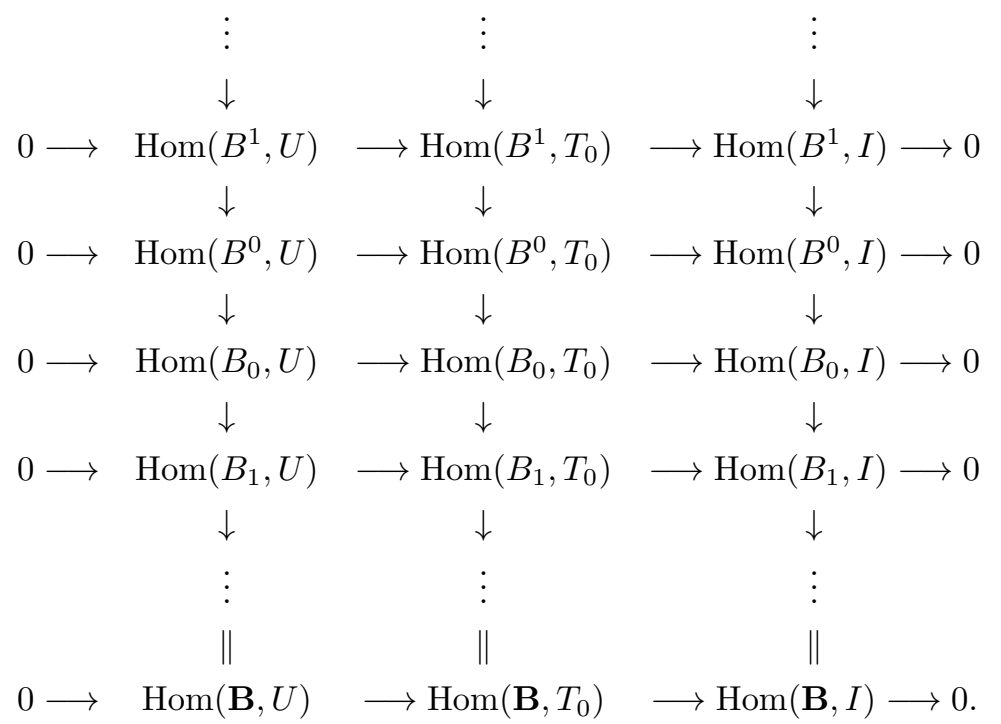

By induction, $\operatorname{Hom}\left(\mathbf{B}, T_{0}\right)$ and $\operatorname{Hom}(\mathbf{B}, I)$ are exact, hence $\operatorname{Hom}(\mathbf{B}, U)$ is exact by [8, Theorem 6.10]. Therefore, $G$ is Gorenstein $\pi[T]$-projective.

It is worthy to mention that the notion of $T$-injectivity ( $T$-projectivity) is different from the notion of an $M$-injective ( $M$-projective) module in [2].

Corollary 2.5. Let $R$ be a $T$-cocoherent ring and $G \in \operatorname{Gen} T$ be a module. Then the following assertions are equivalent:

(1) $G$ is Gorenstein $\pi[T]$-projective;

(2) There is an exact sequence $0 \rightarrow G \rightarrow B^{0} \rightarrow B^{1} \rightarrow \cdots$ of modules, where every $B^{i}$ is $\pi[T]$-projective;

(3) There is a short exact sequence $0 \rightarrow G \rightarrow M \rightarrow I \rightarrow 0$ of modules, where $M$ is $\pi[T]$-projective and $I$ is Gorenstein $\pi[T]$-projective.

Proof. (1) $\Rightarrow(2)$ and (1) $\Rightarrow(3)$ follow from definition.

$(2) \Rightarrow(1)$ For module $G \in \operatorname{Gen} T,[6$, Proposition 2.1] implies that $\operatorname{Gen} T=$ Pres $^{\infty} T$. So, there is an exact sequence

$$
\cdots \longrightarrow T_{1} \longrightarrow T_{0} \longrightarrow G \longrightarrow 0
$$

where any $T_{i}$ is $\pi[T]$-projective by Remark 2.2. Thus, the exact sequence

$$
\cdots \longrightarrow T_{1} \longrightarrow T_{0} \longrightarrow B^{0} \longrightarrow B^{1} \longrightarrow \cdots
$$

of $\pi[T]$-projective modules exists, where $G=\operatorname{ker}\left(B^{0} \rightarrow B^{1}\right)$. Therefore, $G$ is Gorenstein $\pi[T]$-projective, by Theorem 2.4 . 
$(3) \Rightarrow(2)$ Assume that the exact sequence

$$
0 \longrightarrow G \longrightarrow M \longrightarrow I \longrightarrow 0
$$

exists, where $M$ is $\pi[T]$-projective and $I$ is Gorenstein $\pi[T]$-projective. Since $I$ is Gorenstein $\pi[T]$-projective, there is an exact sequence

$$
0 \rightarrow I \rightarrow C^{0} \rightarrow C^{1} \rightarrow \cdots
$$

where every $C^{i}$ is $\pi[T]$-projective. Assembling the sequences (1) and (2), we get the exact sequence

$$
0 \rightarrow G \rightarrow M \rightarrow C^{0} \rightarrow C^{1} \rightarrow \cdots,
$$

where $M$ and every $C^{i}$ are $\pi[T]$-projective, as desired.

Proposition 2.6. For any module $G \in \mathrm{Gen} T$, the following statements hold.

(1) If $G$ is Gorenstein $\pi[T]$-projective, then $\mathcal{E}_{T}^{i}(G, U)=0$ for all $i>0$ and every module $U \in \mathrm{F}$.Copres ${ }^{1} T$ with T.i.dim $(U)<\infty$.

(2) If $0 \rightarrow N \rightarrow G_{n-1} \rightarrow \cdots \rightarrow G_{0} \rightarrow G \rightarrow 0$ is an exact sequence of modules where every $G_{i}$ is a Gorenstein $\pi[T]$-projective and $G_{i} \in \mathrm{Gen} T$, then $\mathcal{E}_{T}^{i}(N, U)=\mathcal{E}_{T}^{n+i}(G, U)$ for any $i>0$ and any module $U \in$ F.Copres ${ }^{1} T$ with T.i.dim $(U)<\infty$.

Proof. (1) Let $G$ be a Gorenstein $\pi[T]$-projective module, and T.i.dim $(U)=m<$ $\infty$. Then by hypothesis, the following $\pi[T]$-projective resolution of $G$ exists:

$$
0 \rightarrow G \rightarrow B^{0} \rightarrow \cdots \rightarrow B^{m-1} \rightarrow N \rightarrow 0 .
$$

By Remark 2.2, $\mathcal{E}_{T}^{i}\left(B_{j}, U\right)=0$ for every $i>0$ and every $0 \leq j \leq m-1$. Since T.i. $\operatorname{dim}(U)=m$, we deduce that $\mathcal{E}_{T}^{i}(G, U) \cong \mathcal{E}_{T}^{m+i}(N, U)=0$.

(2) Setting $G_{n}=N$ and $K_{j}=\operatorname{ker}\left(G_{j} \rightarrow G_{j-1}\right)$, for every $0 \leq j \leq n$, the short exact sequence $0 \rightarrow K_{j} \rightarrow G_{j} \rightarrow K_{j-1} \rightarrow 0$ exists. Thus by (1), the induced exact sequences

$$
0=\mathcal{E}_{T}^{r}\left(G_{j}, U\right) \rightarrow \mathcal{E}_{T}^{r}\left(K_{j}, U\right) \rightarrow \mathcal{E}_{T}^{r+1}\left(K_{j-1}, U\right) \rightarrow \mathcal{E}_{T}^{r+1}\left(G_{j}, U\right)=0
$$

exists and so $\mathcal{E}_{T}^{r}\left(K_{j}, U\right) \cong \mathcal{E}_{T}^{r+1}\left(K_{j-1}, U\right)$, for every $r \geq 0$. Since $K_{n-1}=N$, we have

$$
\mathcal{E}_{T}^{n+i}(G, U) \cong \mathcal{E}_{T}^{n+i-1}\left(K_{0}, U\right) \cong \cdots \cong \mathcal{E}_{T}^{i}(N, U),
$$

as desired.

Next, we study the Gorenstein $\pi[T]$-projectivity of modules on $T$-cocoherent rings, in short exact sequences. 
Proposition 2.7. Let $R$ be $T$-cocoherent and consider the exact sequence $0 \rightarrow$ $N \rightarrow B \rightarrow G \rightarrow 0$, where $B$ is $\pi[T]$-projective. Then GT-pd $(G) \leq \mathrm{GT}-\operatorname{pd}(N)+1$. In particular, if $G$ is Gorenstein $\pi[T]$-projective, so is $N$.

Proof. We shall show that GT-pd $(G) \leq \mathrm{GT}-\operatorname{pd}(N)+1$. In fact, we may assume that $\operatorname{GT}-\operatorname{pd}(N)=n<\infty$. Then, by definition, $N$ admits a Gorenstein $\pi[T]$ projective resolution:

$$
0 \rightarrow B_{n} \rightarrow B_{n-1} \rightarrow \cdots \rightarrow B_{0} \rightarrow N \rightarrow 0 .
$$

Assembling this sequence and the short exact sequence $0 \rightarrow N \rightarrow B \rightarrow G \rightarrow 0$, the following commutative diagram is obtained:

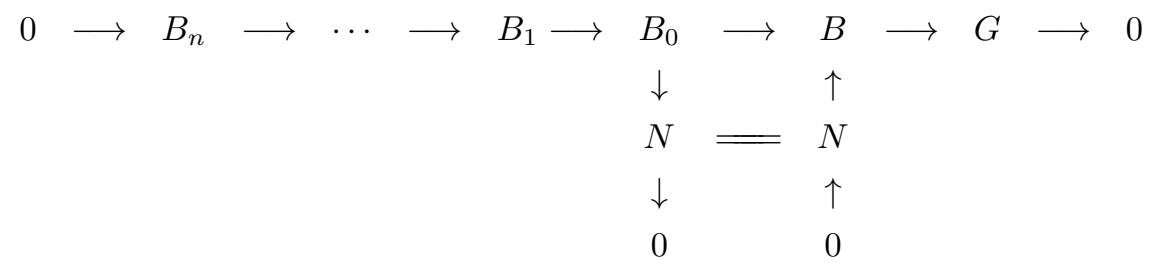

which shows that GT-pd $(G) \leq n+1$. The particular case follows from Corollary 2.5 .

Proposition 2.8. Let $R$ be a T-cocoherent ring and $0 \rightarrow N \rightarrow G \rightarrow B \rightarrow 0$ be an exact sequence, where $N, B \in \mathrm{Gen} T$. If $N$ is Gorenstein $\pi[T]$-projective and $B$ is $\pi[T]$-projective, then $G$ is Gorenstein $\pi[T]$-projective.

Proof. Since $N$ is Gorenstein $\pi[T]$-projective, by Corollary 2.5, there exists an exact sequence of $0 \rightarrow N \rightarrow B^{\prime} \rightarrow K \rightarrow 0$, where $B^{\prime}$ is $\pi[T]$-projective and $K$ is Gorenstein $\pi[T]$-projective. Now, we consider the following diagram:

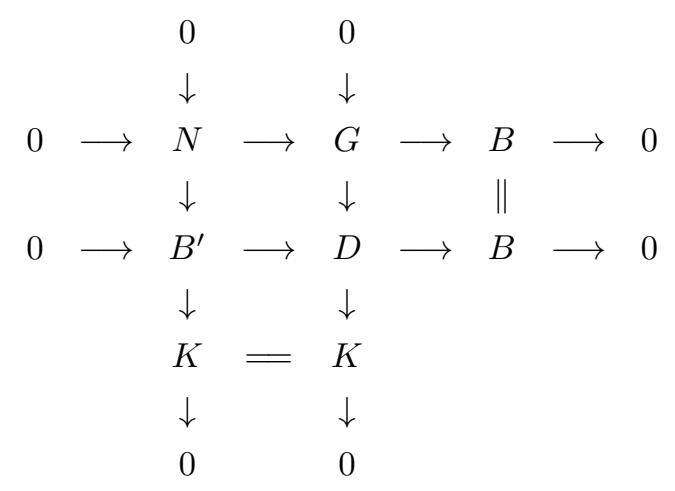

The exactness of the middle horizontal sequence with $B$ and $B^{\prime}, \pi[T]$-projective, implies that $D$ is $\pi[T]$-projective. Hence from the middle vertical sequence and Corollary 2.5, we deduce that $G$ is Gorenstein $\pi[T]$-projective. 


\section{Gorensetein $\pi[T]$-projective modules on $T$-cocoherent rings}

This section is devoted to $T$-cocoherent rings over which every module is Gorenstein $\pi[T]$-projective.

Lemma 3.1. Let $T$ be a tilting module and $G \in G e n T$. Then, $G \in C o g e n T$.

Proof. Let $G \in G e n T$. Then, the short exact sequence $0 \rightarrow K \rightarrow T^{(I)} \rightarrow G \rightarrow 0$ exists. We have $K \subseteq T^{(I)} \subseteq T^{I}$. So, $K \in \operatorname{Cogen} T$. By [6, Proposition 2.1], Cogen $T=$ Copres $^{\infty} T$, since $T$ is tilting. Thus by Lemma 2.3, $G \in$ Copres $^{m} T$, and hence $G \in \operatorname{Cogen} T$.

Proposition 3.2. Let $R$ be a ring. The following assertions are equivalent:

(1) Every module belong GenT, is Gorenstein $\pi[T]$-projective;

(2) The ring satisfies the following two conditions:

(i) Every $T$-injective module is $\pi[T]$-projective.

(ii) $\mathcal{E}_{T}^{1}(N, U)=0$ for any $N \in$ Gen $T$ and any $U \in$ F.Copres $^{n} T$ with T.i.dim $(U)<\infty$.

Proof. $(1) \Rightarrow(2)$ The condition $(i)$ follows from this fact that every $T$-injective module $M$ is Gorenstein $\pi[T]$-projective. So, the following $\pi[T]$-projective resolution of $M$ exists:

$$
0 \rightarrow M \rightarrow B^{0} \rightarrow B^{1} \rightarrow \cdots .
$$

Since $M$ is $T$-injective, $M$ is $\pi[T]$-projective as a direct summand of $B^{0}$. Also, Proposition 2.6(1) and (1) imply that $\mathcal{E}_{T}^{1}(N, U)=0$ for any module $N \in$ GenT and any module $U \in \mathrm{F}$.Copres ${ }^{1} T$ with finite $T$-injective dimension. So the condition (ii) follows.

(2) $\Rightarrow(1)$ Let $G \in \operatorname{Gen} T$. Then by Lemma 3.1, $G \in \operatorname{CogenT}$. So, a AddTresolution $\cdots \rightarrow T_{1} \rightarrow T_{0} \rightarrow G \rightarrow 0$ and a $\operatorname{Prod} T$ - resolution $0 \rightarrow G \rightarrow T^{0} \rightarrow T^{1} \rightarrow$ $\cdots$ of $G$ exists. By Remark 2.2, any $T_{i}$ is $\pi[T]$-projective and any $T^{i}$ is $T$-injective. Hence by (2), every $T^{i}$ is $\pi[T]$-projective. Assembling these resolutions, we get the following exact sequence of $\pi[T]$-projective modules:

$$
\mathbf{B}=\cdots \rightarrow T_{1} \rightarrow T_{0} \rightarrow T^{0} \rightarrow T^{1} \rightarrow \cdots,
$$

where $G=\operatorname{ker}\left(T^{0} \rightarrow T^{1}\right)$. So by $(2)(\mathrm{ii}), \operatorname{Hom}(\mathbf{B}, U)$ is exact for any module $U \in \mathrm{F}$.Copres ${ }^{1} T$ with finite $T$-injective dimension. Hence $G$ is Gorenstein $\pi[T]$ projective.

The next theorem shows that if $R$ is a $T$-cocoherent ring and every $\sigma[T]$-injective module is Gorenstein $\pi[T]$-projective, then every module is Gorenstein $\pi[T]$-projective. 
Theorem 3.3. Let $R$ be a T-cocoherent ring. Then the following are equivalent:

(1) Every module is Gorenstein $\pi[T]$-projective;

(2) Every Gorenstein $\sigma[T]$-injective module is Gorenstein $\pi[T]$-projective;

(3) Every $\sigma[T]$-injective module is Gorenstein $\pi[T]$-projective;

(4) Every T-injective module is $\pi[T]$-projective.

Proof. $(1) \Rightarrow(2)$ This is clear.

$(2) \Rightarrow(3)$ Let $G$ be a $\sigma[T]$-injective module. Every $\sigma[T]$-injective module is Gorenstein $\sigma[T]$-injective (see,[9]). Since $G$ is Gorenstein $\sigma[T]$-injective, we deduce that $G$ is Gorenstein $\pi[T]$-projective by hypothesis.

$(3) \Rightarrow(4)$ Let $G$ be a $T$-injective module. Then $G$ is $\sigma[T]$-injective, and so $G$ is Gorenstein $\pi[T]$-projective by hypothesis. By Corollary 2.5, there exists an exact sequence $0 \rightarrow G \rightarrow B \rightarrow N \rightarrow 0$, where $B$ is $\pi[T]$-projective. Thus the sequence splits. Hence $G$ is $\pi[T]$-projective as a direct summand of $B$.

(4) $\Rightarrow(1)$ Let $G \in$ GenT. Then by Lemma 3.1, there is an exact sequence

$$
0 \longrightarrow G \longrightarrow T^{0} \longrightarrow T^{1} \longrightarrow \cdots,
$$

where any $T^{i}$ is $T$-injective. Then by (5), every $T^{i}$ is $\pi[T]$-projective. Hence Corollary 2.5 completes the proof.

We denote the right $\pi[T]$-projective dimension of any module $M$ by $\pi[T] . p d(M)$, and $\pi[T] . p d(M)=\inf \left\{n: \mathcal{E}_{T}^{n+1}(M, N)=0\right.$ for every $\left.N \in \pi[T]\right\}$.

Example 3.4. Let $R$ be a 1-Gorenstein ring and $0 \rightarrow R \rightarrow E^{0} \rightarrow E^{1} \rightarrow 0$ be the minimal injective resolution of $R$. Then, $\pi[T] \cdot p d\left(E^{0}\right)=\pi[T] \cdot p d\left(E^{1}\right)=0$. Since by [4], $T=E_{0} \oplus E_{1}$ is a tilting module. So, any $E^{i}$ is $\pi[T]$-projective and hence, any $E^{i}$ is Gorenstein $\pi[T]$-projective for $i=0,1$.

Definition 3.5. We define the global $\pi[T]$-projective dimension of any ring $R$ to be:

$$
g l . \pi[T] . p d(R)=\sup \{\pi[T] \cdot p d(M) \mid M \text { is a module }\} .
$$

Clearly, every $\pi[T]$-projective module is Gorenstein $\pi[T]$-projective. But the converse is not true in general. We finish this paper with the following theorem which determines a sufficient condition under which the converse holds.

Theorem 3.6. If gl. $\pi[T] . p d(R)<\infty$, then every Gorenstein $\pi[T]$-projective module is $\pi[T]$-projective. 
Proof. Suppose that $g l \cdot \pi[T] \cdot \operatorname{pd}(R)=m<\infty$, and $G$ is a Gorenstein $\pi[T]$ projective module. If $m=0$, then $\mathcal{E}_{T}^{1}(M, N)=0$ for any $N \in \pi[T]$, and hence $G$ is $\pi[T]$-projective. For $m \geq 1$, since $G$ is Gorenstein $\pi[T]$-projective, there exists an exact sequence $0 \rightarrow G \rightarrow B^{0} \rightarrow B^{1} \rightarrow \cdots$ with each $B^{i}$ is $\pi[T]$-projective. Let $L=\operatorname{coker}\left(B^{m-2} \rightarrow B^{m-1}\right)$. Then

$$
0 \longrightarrow G \longrightarrow B^{0} \longrightarrow B^{1} \longrightarrow \cdots \longrightarrow B^{m-2} \longrightarrow B^{m-1} \longrightarrow L \longrightarrow 0
$$

is exact, and hence $G$ is $\pi[T]$-projective since $\pi[T] \cdot \operatorname{pd}(L) \leq m$.

Acknowledgement. The author would like to thank the referee for the valuable suggestions and comments.

\section{References}

[1] M. Amini and F. Hasani, Copresented dimension of modules, Iran. J. Math. Sci. Inform., 14(2) (2019), 153-157.

[2] F. W. Anderson and K. R. Fuller, Rings and Categories of Modules, Graduate Texts in Mathematics, 13, Springer-Verlag, New York-Heidelberg, 1974.

[3] S. Bazzoni, A characterization of n-cotilting and n-tilting modules, J. Algebra, 273(1) (2004), 359-372.

[4] E. E. Enochs and O. M. G. Jenda, Relative Homological Algebra, De Gruyter Expositions in Mathematics, 30, Walter de Gruyter \& Co., Berlin, 2000.

[5] S. Glaz, Commutative Coherent Rings, Lecture Notes in Mathematics, 1371, Springer-Verlag, Berlin, 1989.

[6] M. J. Nikmehr and F. Shaveisi, Relative T-injective modules and relative T-flat modules, Chin. Ann. Math. Ser. B, 32(4) (2011), 497-506.

[7] M. J. Nikmehr and F. Shaveisi, T-dimension and $\left(n+\frac{1}{2}, T\right)$-projective modules, Southeast Asian Bull. Math., 36 (2012), 113-123.

[8] J. J. Rotman, An Introduction to Homological Algebra, Second edition, Universitext, Springer, New York, 2009.

[9] F. Shaveisi, M. Amini and M. H. Bijanzadeh, Gorenstein $\sigma[T]$-injectivity on T-coherent rings, Asian-Eur. J. Math., 8(4) (2015), 1550083 (9 pp).

[10] R. Wisbauer, Foundations of Module and Ring Theory, Algebra, Logic and Applications, 3, Gordon and Breach Science Publishers, Philadelphia, PA, 1991.

[11] W. Xue, On n-presented modules and almost excellent extensions, Comm. Algebra, 27(3) (1999), 1091-1102. 
[12] Z. M. Zhu and J. L. Chen, FCP-projective modules and some rings, J. Zhejiang Univ. Sci. Ed., 37(2) (2010), 126-130.

\author{
M. Amini \\ Department of Mathematics \\ Faculty of Science \\ Payame Noor University \\ Tehran, Iran \\ e-mail: mostafa.amini@pnu.ac.ir
}

